\title{
Pattern Synthesis using Genetic Algorithm for Low Sidelobe Levels
}

\author{
V. Rajya Lakshmi \\ Dept of ECE \\ Anil Neerukonda Institute of Technology and \\ Sciences, Visakhapatnam, Andhra Pradesh, India
}

\author{
G. S. N. Raju \\ Dept of ECE \\ Andhra University College of Engineering, \\ Visakhapatnam, Andhra Pradesh, India
}

\begin{abstract}
For pattern synthesis of arrays, it is required to find out appropriate weighting vectors to yield a desired radiation pattern. It is well known that several methods for above purpose are available in literature. It is an established fact that when an attempt is made to reduce the first sidelobe level, the beamwidth increases. However, it is of interest in the present work to synthesize an array for a specified first side lobe level without deteriorating the beamwidth. For this purpose, well known analytical technique for array synthesis to control side lobe level, Dolph Chebyshev method is used with the application of WIPL-D Microwave EM software, and the excitation levels are found out. They are also found out using Genetic Algorithm (GA). The weighting vectors are compared in the form of a Table. Using these vectors, the patterns are generated for arrays of different elements. The resultant patterns are compared for the above methods.
\end{abstract}

\section{Keywords}

Genetic algorithm, Dolph Chebyshev method, Side lobe level, Array synthesis, WIPL-D Microwave.

\section{INTRODUCTION}

Array synthesis is one of the most important problems in all communication and radar applications. For this purpose, Chebyshev and Taylor methods are well established and widely used [1-2]. However, they are applied for uniformly spaced linear arrays containing isotropic elements. Moreover, Chebyshev method [3] leads the main beam and a set of sidelobes at equal height. On the other hand, Taylor's method results in the main beam with one set of sidelobes at equal height and another set with decaying sidelobes.

In most of the applications, the high first side lobe level introduces EMI problems and hence it should be lowered without disturbing the beam width. [4-5].During the last one decade, numerical methods are becoming popular to synthesize the patterns to meet the specifications demanded by the user [67]. Such methods are easily extendable to linear, non linear, circular and elliptical arrays etc. These methods include linear optimization methods, non linear optimization, adaptive methods [8-9] dynamic programming [10], downhill, Powell's, conjugate gradient and genetic algorithm etc. Although, most of the above methods are widely applied, simulated annealing, GA [11-13] has the characteristics of universal technique. In fact, it is robust and lesser number of assumptions in the search arena. It is potential in obtaining the solution of complex problems in the design of antennas [14-18]. Some of the researchers [19-20] applied RGA (Real coded Genetic Algorithm) with an aim to reduce sidelobes of asymmetrical linear antenna. In the works presented above, it is evident that when the first side lobe is reduced, null to null beamwidth is enhanced. It means, directivity of the array is disturbed.

For this purpose, the Dolph Chebyshev excitation coefficients are obtained and the patterns are presented for different arrays. In order to reduce the first sidelobe further, GA is applied to find out excitation coefficients. Introducing these excitation levels to each element of the array the radiation patterns are numerically computed for different arrays. The resultant patterns are compared with those of Dolph Chebyshev. The patterns of Dolph Chebyshev are obtained using WIPL-D microwave EM software.

\section{ARRAY SYNTHESIS}

In antenna design, it is required to obtain narrowest main beam width along with low side lobe levels. In uniform linear arrays the array elements are excited uniformly. It is found to give a side lobe level of $-13.5 \mathrm{~dB}$ with a beam width which varies with number of elements in the array. On the other hand in binomial arrays, the radiating elements of the array are excited in accordance with the binomial coefficients. The patterns of these arrays are found to contain no side lobes with enhanced beam width from that of uniform linear array.

Dolph Chebyshev method provides a compromise between uniform and binomial arrays. It provides a means of determining suitable polynomials which give excitation coefficients to obtain required patterns. It is a method which yields radiation pattern containing one main beam and sidelobes with the same levels. This is achieved by finding spacing of nulls. The recursion formula for Chebyschev's polynomial is

$\mathrm{T}_{\mathrm{m}}(z)=2 z \mathrm{~T}_{\mathrm{m}-1}(z)-\mathrm{T}_{\mathrm{m}-2}(z)$

Each polynomial can also be computed using

$$
\begin{array}{lr}
\mathrm{T}_{\mathrm{m}}(\mathrm{z})=\cos \left(\mathrm{m} \cos ^{-1}(z)\right) & -1 \leq z \leq+1 \\
\mathrm{~T}_{\mathrm{m}}(\mathrm{z})=\cos \left(\mathrm{m} \cosh ^{-1}(z)\right) & z<-1, z>+1
\end{array}
$$

The array factor of linear isotropic elements is

$$
\begin{aligned}
& (\mathrm{AF})_{2 \mathrm{M}(\text { even })}=\sum_{\mathrm{n}=1}^{\mathrm{M}} \mathrm{a}_{\mathrm{n}} \cos [(2 \mathrm{n}-1) \mathrm{u}] \\
& (\mathrm{AF})_{2 \mathrm{M}+1 \text { (odd) }}=\sum_{\mathrm{n}=1}^{\mathrm{M}+1} \mathrm{a}_{\mathrm{n}} \cos [(2 \mathrm{n}-1) \mathrm{u}]
\end{aligned}
$$

Where $u=\pi d / \lambda \cos \theta$ 
Since the array factor of an even or odd number of elements is a summation of cosine terms whose form is the same as the Tschebyschev polynomials the unknown coefficients of the array factor can be determined by equating the series representing the cosine terms of the array factor to the appropriate Tschebyschev polynomial.

In the present work, the array designer in WIPL-D software is applied to compute array coefficients. It is an add on tool of the software which is useful for automated antenna array design and also analysis. It is based on full 3D EM models of individual antenna elements [21]. With 3D EM model containing a generic model of the array to be analyzed is created using WIPL-D. Once a model is prepared, array designer is activated. Its main purpose is to import full 3D EM model with either voltage or current generators connected to the corresponding ports into the schematic.

The tool is applied for Dolph Chebyshev method to obtain the excitation coefficients of the array.SLR is chosen at $-35 \mathrm{~dB}$ in the present case. The arrays of 20, 40,60, 100 isotropic elements are considered to generate the radiation patterns. Moreover, GA is found to have a large number of numerical applications in real world problems encountered in Engineering and Computer science. GA is useful to optimize the solution of any given problem and it is applied successfully in the present context. One of the most successful applications of GA is pattern synthesis of array antennas. It is evident from the literature, and practical experiences, low sidelobe antenna arrays are become very important in high performance electronic systems in communication and radars to overcome the problems of heavy clutter, jamming and interference.

\section{GENETIC ALGORITHM}

Genetic algorithm is a global search algorithm and works on the mechanics of natural selection and genetics. It is used in computing to optimize the solutions of any given problem. It has wide application in every system that exists in nature. For example, chip design, antenna design, agricultural production, CNC machines, design of bridges, phased arrays design etc.[2224].The characteristics of Genetic algorithm are given below.

$>$ It optimizes radiation pattern by continuous or discrete parameters

$>$ It doesn't require derivative information

$>$ It searches simultaneously from a wide range of sample space.

$>$ It works with large number of constrained variables.

$>$ It is best suited for parallel computers.

$>$ Optimization is done with numerically generated data or analytical data or empirical data by encoding parameters.

GA came into existence from Darwin's theory of revolution. It is a powerful evolutionary computation method, and has importance in artificial intelligence also. The Algorithm begins with a set of solutions (represented by chromosomes) called population. Solutions from one population are taken and used to form a new population. This is motivated by a hope, that the new population will be better than the old one. Solutions which are then selected to form new solutions (offspring) are selected according to their fitness - the more suitable they are the more chances they have to reproduce. This is repeated until some condition (for example number of populations or improvement of the best solution) is satisfied. For the sake of the clarity of readers, the outline of the Genetic algorithm is given below.

1) [Start] Generate random population of $n$ chromosomes (suitable solutions for the problem)

2) [Fitness] Evaluate the fitness $f(x)$ of each chromosome $x$ in the population

3) [New population] Create a new population by repeating following steps until the new population is complete

a. [Selection] Select two parent chromosomes from a population according to their fitness (the better fitness, the bigger chance to be selected)

b. [Crossover] With a crossover probability cross over the parents to form new offspring (children). If no crossover was performed, offspring is the exact copy of parents.

c. [Mutation] With a mutation probability mutate new offspring at each locus (position in chromosome).

d. [Accepting] Place new offspring in the new population

4) [Replace] Use new generated population for a further run of the algorithm

5) [Test] If the end condition is satisfied, stop, and return the best solution in current population

6) [Loop] Go to step 2

Chromosomes are selected from the population to be parents for crossover. The problem is how to select these chromosomes. According to Darwin's theory of evolution the best ones survive to create new offspring. There are many methods in selecting the best chromosomes. Examples are roulette wheel selection, Boltzmann selection, tournament selection, rank selection, steady state selection and some others. From the genetic algorithm outline, the crossover and mutation are the most important parts of the genetic algorithm. The performance is influenced mainly by these two operators.

Crossover operates on selected genes from parent chromosomes and creates new offspring. The simplest way is to choose randomly some crossover point and copy everything before this point from the first parent and then copy everything after the crossover point from the other parent. After a crossover is performed, mutation takes place. Mutation is intended to prevent falling of all solutions in the population into a local optimum of the solved problem. In case of binary encoding, we can switch a few randomly chosen bits from 1 to 0 or from 0 to 1 .If mutation is performed; one or more parts of a chromosome are changed. If mutation probability is $100 \%$, whole chromosome is changed, if it is $0 \%$, nothing is changed.

The fitness function or object function is used to assign a fitness value to each of the individuals in the GA population the fitness function is the only connection between the physical problem being optimized and the genetic algorithm. Genetic algorithms are very useful for many electromagnetic optimization problems. 


\section{ARRAY SYNTHESIS AND GA}

The linear array is one of the most commonly used array structure in many different applications owing to its simplicity and beam shaping property .In a conventional linear array, elements are uniformly spaced with half wavelength inter element spacing and uniformly excited. In this paper, amplitude only pattern synthesis is used and main aim is to determine the amplitude levels to produce the lowest possible sidelobe level.

The radiating elements in the array of present interest are considered to be point sources spaced $d=\lambda / 2$ apart. A symmetric linear array is shown in Figure 1.

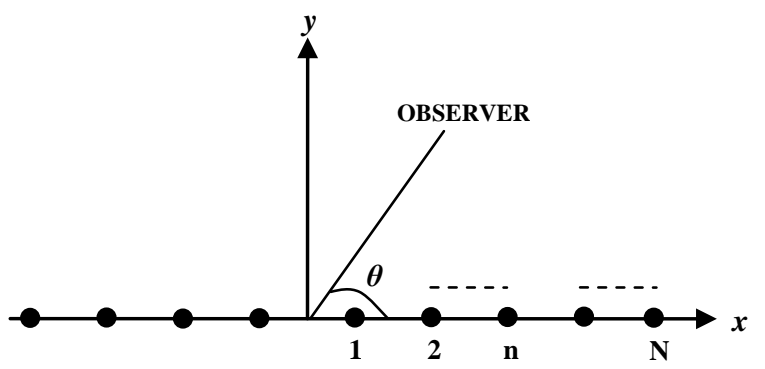

Figure 1: Geometry of 2N element Linear Array.

Mathematically, array factor of a $2 \mathrm{~N}$ element array is given by [25]

$$
\mathrm{E}(\theta)=2 \sum_{\mathrm{n}=1}^{\mathrm{N}} \mathrm{A}_{\mathrm{n}} \mathrm{e}^{\mathrm{j} \varphi_{\mathrm{n}}} \cos \left[\mathrm{k}(\mathrm{n}-0.5) \mathrm{d}\left(\mathrm{u}-\mathrm{u}_{0}\right)\right]
$$

Here, $\mathrm{k}=$ wave number $=2 \pi / \lambda,=\lambda$ wave length.

$$
\begin{aligned}
& A_{n}=\text { Amplitude of the nth element. } \\
& \varphi_{n}=\text { phase of the } n \text {th element. } \\
& d=\text { spacing between the radiating elements. } \\
& \theta=\text { angle between the line of observer and broadside. } \\
& \theta_{0}=\text { scan angle, } u=\sin \theta, u_{0}=\sin \theta_{0}
\end{aligned}
$$

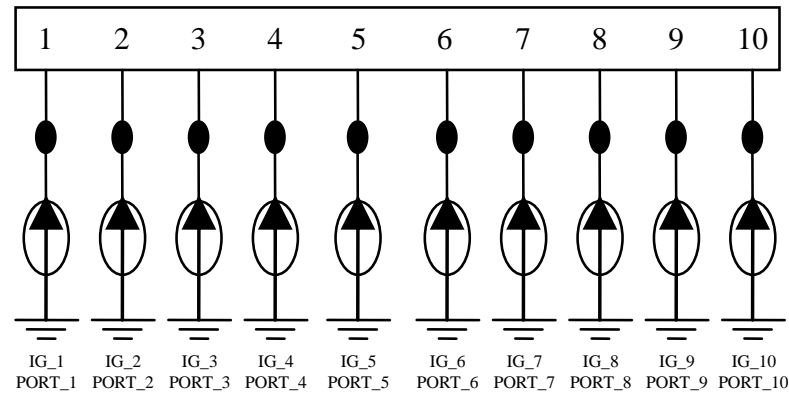

\section{Figure 2: WIPL D microwave Schematic of the half of the} array of 20 elements.

The fitness function associated with this array is the maximum Side Lobe Level of its associated radiation field pattern to be minimized. The general form of the fitness function is given by

Fitness $=\operatorname{Max} \frac{\left(20 \log _{10}(|\mathrm{E}(\theta)|)\right)}{(\max |\mathrm{E}(\theta)|)}-\pi / 2 \leq \theta \leq \pi / 2, \theta \neq 0^{\circ}$

\section{RESULTS}

The schematic of the half of the symmetric linear array of 20 elements in WIPL-D is shown in Figure 2.The remaining half of the array is its mirror image only. The generators are attached to each element in the array. In the present work, inter element spacing is assumed to be $\lambda / 2$ and phase of the excitation is zero degrees. Using Array designer module, the 20 element array is designed for $-35 \mathrm{~dB}$.

The computed amplitude coefficients for 20 element array are tabulated in Table.1.The Real coded genetic algorithm is applied on the same array for less than $-35 \mathrm{~dB}$.The computed coefficients are also tabulated in the same table.

The numerically computed patterns of Dolph Chebyshev method and GA for $20,40,60,100$ elements with $0^{\circ}$ scan angle are presented in Figures [3-6].For a steering angle of $45^{\circ}$ the pattern

\begin{tabular}{|c|c|c|}
\hline S. No & $\begin{array}{c}\text { Chebyshev Amplitude } \\
\text { Excitation }\end{array}$ & $\begin{array}{c}\text { Amplitude Excitation } \\
\text { Using GA }\end{array}$ \\
\hline 1 & 0.1935 & 0.8175 \\
\hline 2 & 0.2180 & 0.4538 \\
\hline 3 & 0.3215 & 0.8034 \\
\hline 4 & 0.4389 & 0.4317 \\
\hline 5 & 0.5636 & 0.3395 \\
\hline 6 & 0.6874 & 0.9427 \\
\hline 7 & 0.8012 & 0.6665 \\
\hline 8 & 0.8961 & 0.6692 \\
\hline 9 & 0.9643 & 0.3763 \\
\hline 10 & 1.0000 & 0.5830 \\
\hline 11 & 1.0000 & 0.5830 \\
\hline 12 & 0.9643 & 0.3763 \\
\hline 13 & 0.8961 & 0.6692 \\
\hline 14 & 0.8012 & 0.6665 \\
\hline 15 & 0.6874 & 0.9427 \\
\hline 16 & 0.5636 & 0.3395 \\
\hline 17 & 0.4389 & 0.4317 \\
\hline 18 & 0.3215 & 0.8034 \\
\hline 19 & 0.2180 & 0.4538 \\
\hline 20 & 0.1935 & 0.8175 \\
\hline
\end{tabular}
of array containing the 100 number of elements are presented in 7.The amplitude distribution ,A(x) obtained using Dolph Chebyshev technique is presented in Figure 8. The distribution obtained with GA is presented in Figure 9.

Table 1: Excitation levels of 20 Element array. 


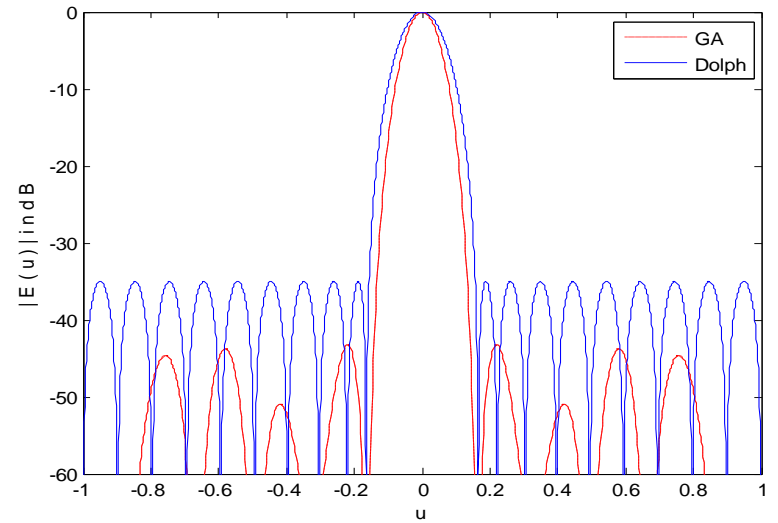

Figure 3: Patterns of 20 element array with Dolph Chebyshev method and GA

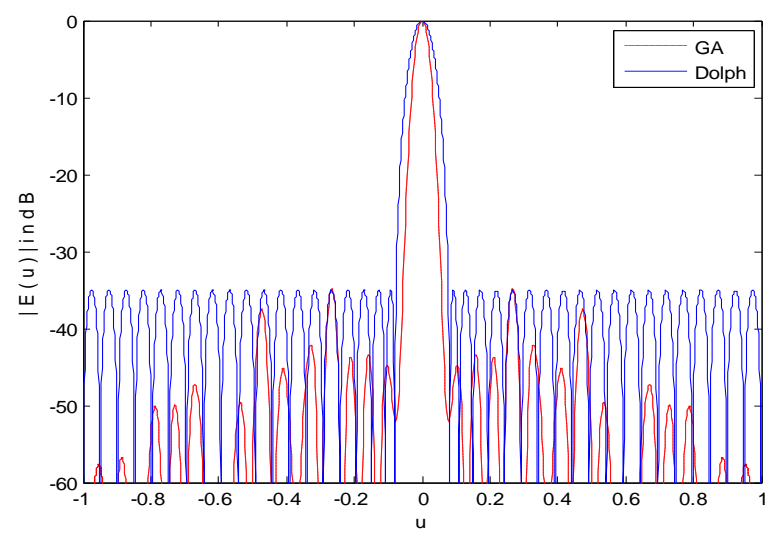

Figure 4: Patterns of 40 element array with Dolph Chebyshev method and GA

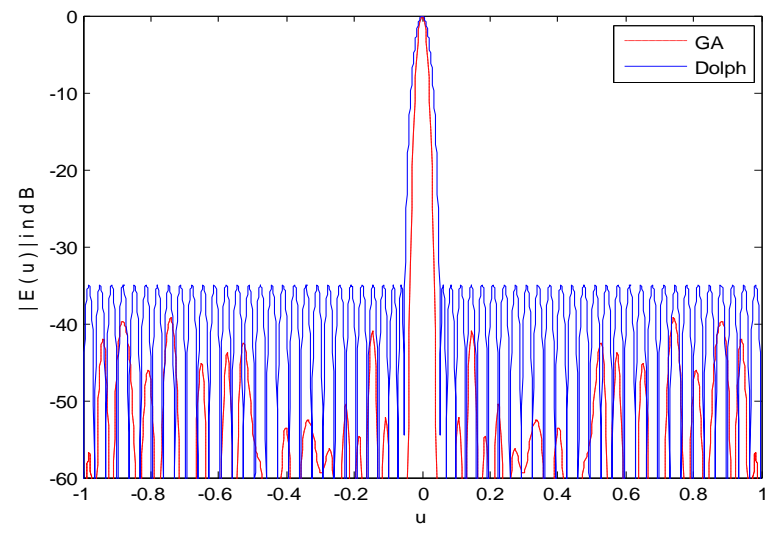

Figure 5: Patterns of 60 element array with Dolph Chebyshev method and GA

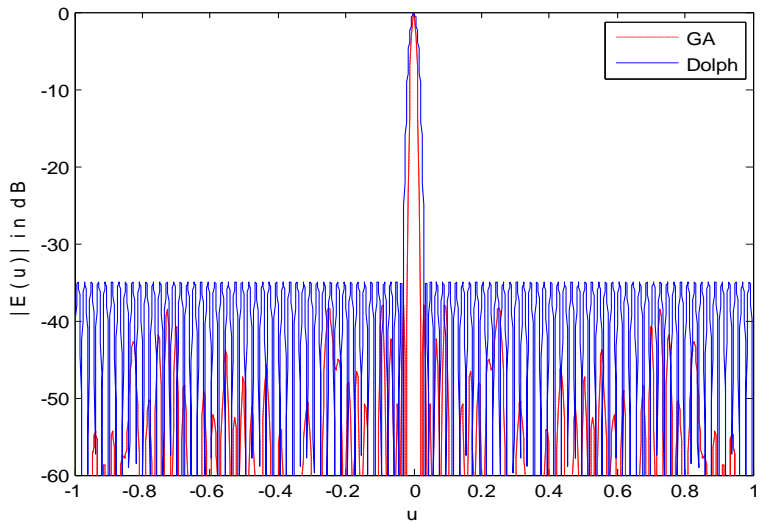

Figure 6: Patterns of 100 element array with Dolph Chebyshev method and GA

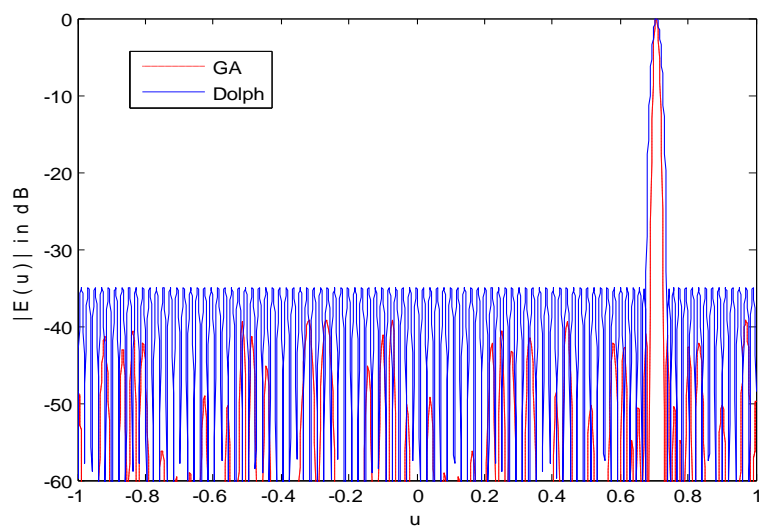

Figure 7: Steered beams of 100 element array with Dolph chebyschev method and GA for $\boldsymbol{\theta}_{0}=\mathbf{4 5}^{\circ}$

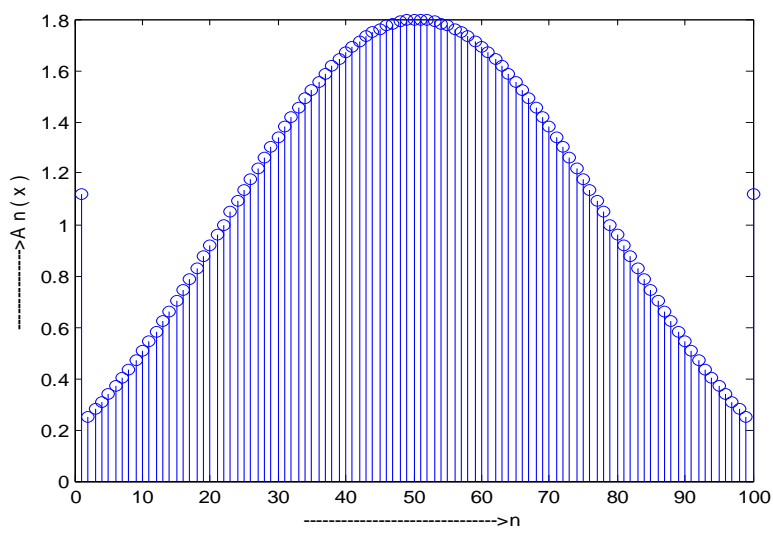

Figure 8: Amplitude distribution of 100 array elements using Dolph Technique. 


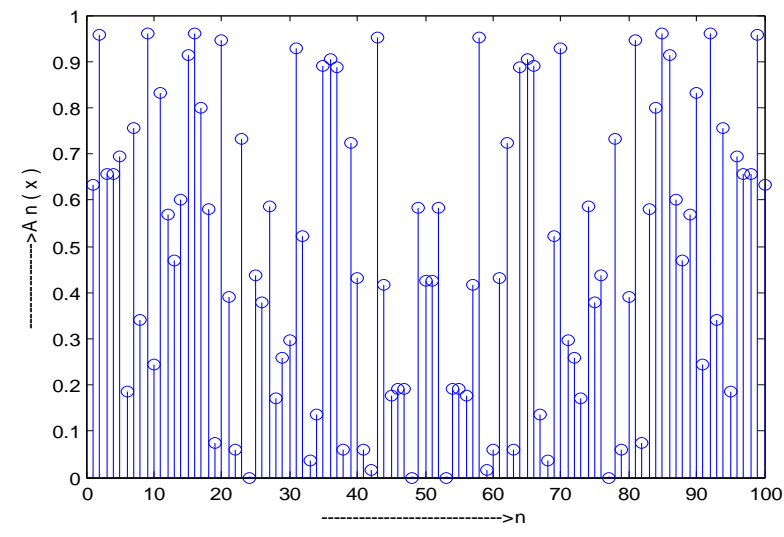

Figure 9: Amplitude distribution of 100 array elements using GA.

\section{CONCLUSION}

The excitation levels of Dolph Chebyshev method are tapered in nature from the centre of the array. However, the excitation levels computed using GA are random in height with no regular taper. But the excitation levels in both the cases are symmetric to the centre of the array. The first sidelobe level in GA method is lower than that of Dolph Chebyshev and at the same time, null to null beamwidth is also marginally reduced. This is true in both scan and non scanned beams. Although the present work is focused on arrays of isotropic radiators, the GA technique can be extended for arrays of practical elements.

\section{REFERENCES}

[1] Raju, G. S. N., 2005. Antennas and propagation, Pearson Education.

[2] Elliot, R. S., 1981. Antenna Theory and Design. PrenticeHall. New Jersey.

[3] Dolph, C. L., June 1946. A current distribution for broadside arrays which optimizes the relationship between beam width and side-lobe level, Proc. IRE 34:335-348.

[4] Taylor, T. T., Jan. 1955. Design of line source antennas for narrow beam widths and low sidelobes, IRE AP Trans. 4.16-28.

[5] Villeneuve, A. T., Oct. 1984. Taylor patterns for discrete arrays, IEEE AP-S Trans. 32(10) 1089-1094.

[6] Y. C. Jiao, W. Y. Wei, L. W. Huang, H. S. Wu, "A new low side lobe pattern synthesis technique for conformal arrays", IEEE Trans. Antenna Propagation. Vol 41, pp 824831, June 1993.

[7] Sim, M. H .Er. S. L., and Koh, S. N., Nov. 1993. "Application of constrained optimization techniques to array pattern synthesis", Signal Processing. Vol 34, pp 323334.

[8] Dufort, E. C., Aug 1989. "Pattern synthesis based on adaptive array theory", IEEE Trans. Antenna Propagat. Vol 37, pp 1011-1018.

[9] Olen, C. A., and Compton Jr. R. T., Oct. 1990. "A numerical pattern synthesis algorithm for arrays", IEE Trans. Antenna Propagat. Vol 30, pp 1666-1676.
[10] Skolnik, M., Nemhauses, G., and Shermas, J., Jan 1964. "Dynamic programming applied to unequally spaced array", IEEE Trans. Antenna and Propogat. Vol 12, pp 3543.

[11] Samii, Y. R., and Michelssen, E., 1999. Electromagnetic Optimization by Genetic Algorithm, John Wiley \& Sons, New York.

[12] Haupt, R. L., Apr. 1995. "An introduction to genetic algorithm for electromagnetics", IEEE Antennas Propogat. Mag. Vol 37, no 2, pp 7-15.

[13] Wei, D., and Michielssen, E., Mar. 1997. "Genetic algorithm optimizations applied to electromagnetic: A review", IEEE Trans Antennas and Propogat. Vol. 45, No.3.

[14] Shinizu, M., 1994. "Determining the Excitation coefficients of an array using Genetic Algorithm", IEEE AP-S, International symposium, Seattle, June 19-24, Vol.1, pp530-533.

[15] Tennant, A., Dawood, M., and Anderson, A., February 1994. "Array pattern nulling by Elert position perturbation using a Genetic Algorithm", Electronic letters, Vol 30, No 3 , pp 174-176.

[16] Johnson, J., and Rahmat-Samii, Y., 1994. "Genetic algorithm optimization and its application to antenna design", IEEE AP-S International Symposium, Seattle, June 19-24, Vol. 1, pp 326-329.

[17] Haupt, R., July 1994. "Thinned Arrays using Genetic Algorithm", IEEE Trans Antenna and Propogat. Vol 42, No 7, pp 993-999.

[18] Bahr, M., Boag, A., Michielssen E., and Mittra, R., 1994. “ Design of ultra broadband loaded monopoles", IEEE AP-S International Symposium, Seattle, June 19-24, Vol 2, pp $1290-1293$

[19] Maudal, D., Ghoshal, S. K., Das, S., Bhatttacharjee, S., and Bhattacharjee, A. K., 2010. "Improvement of radiation pattern for linear antenna arrays using GA" International conference on Recent Trends in Informatics, Telecommunication and Computing.

[20] Sudipta Das, Somes Bhattacherjee, Durbadal Mandal, Anup Kumar Bhattacharjee, 2010. "Optimal sidelobe reduction of symmetric linear antenna array using Genetic Algorithm”, 2010 Annual IEE Conference (INDICON).

[21] WIPL-D software manual.

[22] Johnson, J. M., and Samii, Y. R., 1997. "Genetic Algorithm in engineering electromagnetic", IEEE antenna and propagate. Magazine, vol.39, No 4.

[23] Johnson, J. M., and Samii, Y. R., 1996. "Genetic Algorithm optimization for aerospace electromagnetics", IEEE aerospace applications conference proceedings volume 1 , pp 87-102.

[24] Johnson J. M., and Samii, Y. R., 1996. "Genetic Algorithm in electromagnetics", IEEE antenna and Propagat. Society international symposium digest volume 2, Baltimore, MD, July 21-26, pp 1480-1483.

[25] Balanis, C. A., 1997. Antenna Theory Analysis and Design 2nd Edition, John Wiley \& Sons Inc, New York. 